\title{
Protective effects of N(2)-L-alanyl-L-glutamine mediated by the JAK2/STAT3 signaling pathway on myocardial ischemia reperfusion
}

\author{
SHAN LIU, YANG YANG, YAN QIU SONG, JIE GENG and QING LIANG CHEN \\ Tianjin Cardiovascular Institute, Tianjin Chest Hospital, Tianjin 300222, P.R. China
}

Received February 11, 2017; Accepted August 11, 2017

DOI: $10.3892 / \mathrm{mmr} .2018 .8543$

\begin{abstract}
To explore the protective effect of N(2)-L-alanyl-L-glutamine (NLAG) on myocardial ischemia-reperfusion injury (IRI), and observe the influence of NLAG on the Janus activated kinase signal transducer 2 and activator of transcription 3 (JAK2/STAT3) signaling pathway-associated molecules. Wistar rats were randomly divided into three groups: Sham, IRI and NLAG. In the IRI rat model, the cardiac hemodynamics, the maximum rate of left ventricular pressure (+dP/dtmax) and the left ventricular end-diastolic pressure (LVDP) were recorded. Hematoxylin-eosin and Masson staining were used to detect myocardial histological changes. The levels of plasma interleukin (IL)- $1 \beta$ and -6 , tumor necrosis factor (TNF)- $\alpha$, lactase dehydrogenase ( $\mathrm{LDH})$, troponin (cTn)I, creatine kinase (CK), heart type fatty acid binding protein (hFABP), malondialdehyde (MDA) and succinate dehydrogenase (SDH) were determined with ELISA. The protein expression levels of B-cell lymphoma (Bcl)-2, Bcl2-associated X protein (Bax), Caspase-3, JAK2, phosphorylated (p)-JAK2, STAT3 and p-STAT3 were detected by western blot analysis. The IRI model demonstrated notable myocardial injury; myocardial cells were arranged disorderly with some nuclei disappearing, and cardiac muscular fibers were degenerated. Following $60 \mathrm{~min}$ of reperfusion, LVDP, HR and $+\mathrm{dP} / \mathrm{dtmax}$ were $31.3 \pm 4.53 \mathrm{mmHg}, 239.17 \pm 8.45$ beats $/ \mathrm{min}$ and $615.17 \mathrm{mmHg} / \mathrm{sec}$, respectively. Compared with the Sham group, the levels of $\mathrm{LDH}$, cTnI, CK, hFABP release, inflammatory factors (IL-1 $\beta$, IL- 6 and TNF- $\alpha$ ) and oxygen free radical (MDA and $\mathrm{SDH}$ ) levels were increased in
\end{abstract}

Correspondence to: Dr Jie Geng or Dr Qing Liang Chen, Tianjin Cardiovascular Institute, Tianjin Chest Hospital, 261 Taierzhuang South Road, Jinnan, Tianjin 300222, P.R. China

E-mail: chenqingliang1971@163.com

E-mail: liushan19830329@163.com

Key words: N(2)-L-analyl-L-glutamine, myocardial ischemia reperfusion injury, janus activated kinase signal transducer 2 and activator of transcription 3 , mitochondrial function, oxidative stress injury the IRI group. In the NLAG group, myocardial injury was improved, the concentrations of LDH, cTnI, CK, hFABP, IL-1 $\beta$, IL-6, TNF- $\alpha$, MDA were decreased, and SDH release was increased compared with the IRI group. In addition, NLAG significantly increased Bcl-2, JAK2, p-JAK2, STAT3 and p-STAT3 protein expression, and decreased Bax protein expression compared with the IRI group. In conclusion, myocardial ischemia-reperfusion can lead to myocardial cell apoptosis and myocardial injury and NLAG attenuates the IRI-induced mitochondrial oxidative stress injury and apoptosis by activating the JAK2/STAT3 signaling pathway, thus exerting protective effects against IRI.

\section{Introduction}

Ischemic heart disease (IHD) greatly threatens human health leading to arrhythmia, heart failure and even mortality (1). Coronary artery stenosis is the main cause of ischemic heart disease, and cardiopulmonary bypass-caused arrest also induces ischemic heart disease (2). The treatment of ischemic heart disease relies on the recovery of blood supply, but the restored blood supply may trigger myocardial injury, termed ischemia-reperfusion injury (IRI), which contributes to a poor prognosis in IHD patients.

The commonly used drugs for the prevention and treatment of myocardial ischemia include calcium antagonists, $\beta$-receptor blockers and angiotensin-converting enzyme inhibitors, but they are not ideal due to their side effects and safety and ethical issues (3). A previous study demonstrated that the antioxidant N(2)-L-alanyl-L-glutamine (NLAG) is a precursor of intracellular glutathione and has been proved by the US Food and Drug Administration to treat acetaminophen-induced acute liver failure (4). NLAG can directly scavenge, significantly improve the anti-apoptotic ability, and reduce the release of inflammatory factors, chemokines and adhesion molecules $(5,6)$. In addition, NLAG has a protective effect on the kidney and liver against IRI $(7,8)$. However, any protective effects and the underlying mechanism of NLAG on the myocardial IRI remain unclear.

Protein tyrosine kinase/signal transducer and activator of transcription (JAK/STAT) is a signaling transduction pathway that is closely associated with inflammation (9), oxidative stress (10), cell injury and apoptosis (11). Janus activated kinase signal transducer 2 and activator of transcription 3 
(JAK2/STAT3) is an important signal transduction pathway in the JAK/STAT family. Ischemic preconditioning, ischemic post-conditioning and anti-myocardial IRI effects of some drugs are associated with JAK2/STAT3 pathway activation $(12,13)$. The present study established IRI model to investigate the protective effect of NLAG on myocardial IRI, and to observe the effect of NLAG on JAK2/STAT3 signaling pathway-associated molecules.

\section{Materials and methods}

Animals and ethical approval. Adult male Wistar rats (350-450 g; n=30) were obtained from Military Medical Science Academy of the People's Liberation Army (Beijing, China). Animals were housed at a constant temperature $\left(22 \pm 1^{\circ} \mathrm{C}\right)$, with $50 \%$ humidity, and a 12 -h light-dark cycle. The rats had ad libitum access to food and autoclaved water. All animal procedures were approved by the Animal Experiments Ethics Committee of the Military Medical Science Academy of the People's Liberation Army (Beijing, China).

Establishment of the IRI model. Myocardial IRI model was established as has previously been described (14). Rats were anesthetized with intraperitoneal injection of $2 \%$ pentobarbital sodium (cat. no. 57-33-0; $0.2 \mathrm{ml} / 100 \mathrm{~g}$; Sigma-Aldrich; Merck KGaA, Darmstadt, Germany) the tracheotomy was performed between the third and fourth cartilage rings, and rats received mechanical ventilation. The left anterior descending coronary artery (LAD) was ligated by a $7 / 0$ thread inserting $32 \mathrm{~mm}$ below the left auricle root, crossing the myocardium and suturing below the pulmonary artery cone. Both ends of the thread passed through the polyethylene tubule (epidural catheter), which reached the ventricular wall to block coronary blood flow by tightening the ends of the thread. Following that, the tube was clamped with hemostatic forceps. Electrocardiography revealed ST segment elevation and the myocardial tissue below ligature site became darker; following this, hemostatic forceps were released and the LAD blood flow was restored, elevated ST segment was reduced to above 1/2 and the myocardial tissue became gradually red.

Groups and treatments. Rats were randomly divided into three groups: Sham operation (Sham group; $n=10$ ), myocardial ischemia reperfusion (IRI group; $n=10$ ) and NLAG treatment (NLAG group; $n=10$ ). In the Sham group, rats received the tracheotomy alone. In the IRI group, the IRI model was established. In the NLAG group, rats were injected with $150 \mathrm{mg} / \mathrm{kg}$ NLAG (Chongqing Laimei Pharmaceutical Co., Ltd., Chongqing, China) intraperitoneally $30 \mathrm{~min}$ prior to IRI establishment.

Cardiac hemodynamic changes. The hemodynamic parameters of the heart were recorded using the Datex-Ohmeda S/5 Entropy Module (DRE, Inc., Louisville, KY, USA). Left ventricular diastolic pressure (LVDP), heart rate (HR) and the maximum rate of left ventricular pressure $(+\mathrm{dP} / \mathrm{dtmax})$ were recorded before ischemia, at 15, 30, 45 and $60 \mathrm{~min}$ following reperfusion (HR was recorded every $15 \mathrm{~min}$ ).
Preparation and treatment of rat tissues. All rats were anesthetized with pentobarbital sodium (cat. no. 57-33-0; Sigma-Aldrich; Merck KGaA) at $4 \mathrm{~h}$ following IRI. Blood samples $(3 \mathrm{ml})$ were taken from the internal jugular vein and permitted to clot overnight at $4^{\circ} \mathrm{C}$ prior to centrifugation for $15 \mathrm{~min}$ at $1,000 \mathrm{x} \mathrm{g}$ at $4^{\circ} \mathrm{C}$. Serum aliquots were then removed and samples were incubated at $-20^{\circ} \mathrm{C}$ or $-80^{\circ} \mathrm{C}$. The rat myocardial tissues were collected and fixed in neutral formalin or stored in liquid nitrogen.

Hematoxylin-eosin (HE) staining. Heart tissues were fixed in $10 \%$ formaldehyde for $24 \mathrm{~h}$ at room temperature $(\mathrm{pH}=7.2$; cat. no. G2161; Beijing Solarbio Science \& Technology, Co., Ltd., Beijing, China), and then decalcified, dehydrated, permeabilized using xylene $(50 \%$ xylene for $1 \mathrm{~h}$ and $100 \%$ xylene for $2 \mathrm{~h}$ ), embedded in wax and sliced into $5 \mu \mathrm{m}$ thick sections using a microtome. All of the following steps were carried out at room temperature. Sections were then dewaxed using xylene I for $15 \mathrm{~min}$ and then xylene II for $15 \mathrm{~min}$, hydrated with absolute ethanol for $5 \mathrm{~min}, 90 \%$ ethanol for $2 \mathrm{~min}$ and $70 \%$ ethanol for $2 \mathrm{~min}$, mounted with $10 \%$ hematoxylin (cat. no. G1120; Beijing Solarbio Science \& Technology, Co., Ltd.) for $10 \mathrm{~min}$, differentiated with $1 \%$ hydrochloric acid and ethanol for 3-5 sec, stained with $0.5 \%$ eosin (cat. no. G1120; Beijing Solarbio Science \& Technology, Co., Ltd.) for $1 \mathrm{~min}$, dehydrated in alcohol gradients (70\% for $2 \mathrm{sec}, 90 \%$ for $2 \mathrm{~min}$ and absolute ethanol for $5 \mathrm{~min}$ ) and xylene, cleared and then mounted. Using a light microscope (magnification, x200; Leica DM 4000B; Leica Microsystems, Inc., Buffalo Grove, IL, USA), pathological changes of myocardial tissues were observed.

Masson staining. Heart tissues were fixed using 10\% formaldehyde for $24 \mathrm{~h}$ at room temperature ( $\mathrm{pH}=7.2$; cat. no. $\mathrm{G} 2161$; Beijing Solarbio Science \& Technology, Co., Ltd.), decalcified, dehydrated, permeabilized using xylene (50\% xylene for $1 \mathrm{~h}$ and $100 \%$ xylene for $2 \mathrm{~h}$ ), embedded in wax and then sliced into $5 \mu \mathrm{m}$ thick sections using a microtome. All the following steps were carried out at room temperature. The tissues were routinely deparaffinized and rehydrated in accordance. Weigert's hematoxylin (5\%; cat. no. G1340; Beijing Solarbio Science \& Technology, Co.,Ltd.) was used to dye the cell nucleus for $5 \mathrm{~min}$. Following rinsing with distilled water three times, the sections were stained using 0.7\% Masson-Ponceau-acid fuchsin solution (cat. no. G1340; Beijing Solarbio Science \& Technology, Co., Ltd.) for $10 \mathrm{~min}$. Samples were then rinsed in $2 \%$ glacial acetic acid and differentiated in phosphomolybdic acid for $4 \mathrm{~min}$. The sections were directly stained with $2 \%$ aniline blue dye solution (cat. no. G1340; Beijing Solarbio Science \& Technology, Co., Ltd.). Following dehydrating with ethanol series, clearing with xylene and mounting with neutral resins, digital images were captured using a light microscope (magnification, x200; Leica DM 4000B; Leica Microsystems, Inc.) to analyze fiber formation in the early callus.

ELISA assay. Protein expression changes of markers of myocardial injury [lactase dehydrogenase (LDH; cat. no. SEB864Ra; Uscn Life Sciences, Inc., Wuhan, China), troponin I (cTnI; cat. no. SEA478Ra; Uscn Life Sciences, Inc.), creatine kinase (CK; cat. no. SEA109Ra; Uscn Life Sciences, Inc.) and heart type 

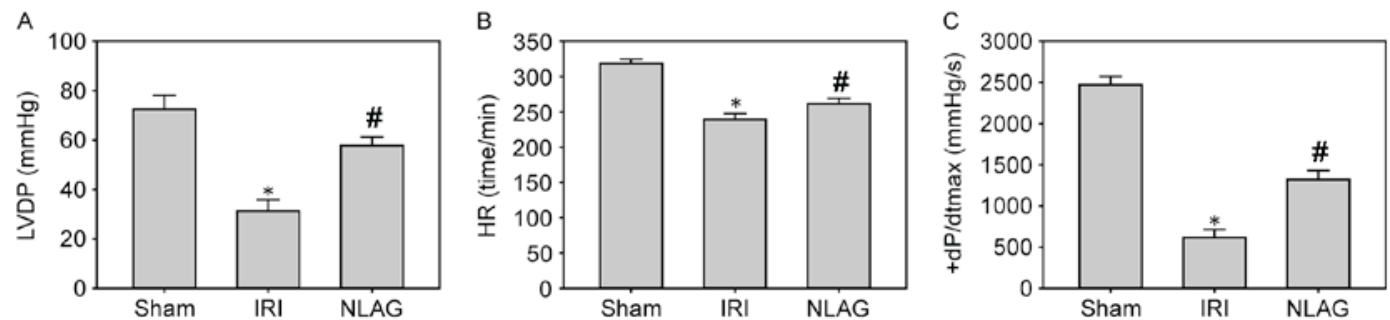

Figure 1. Effect of NLAG on hemodynamic parameters of the rat heart. (A) LVDP, (B) HR and (C) $+\mathrm{dP} / \mathrm{dtmax}$. Data are presented as the mean \pm standard deviation. ${ }^{*} \mathrm{P}<0.05$ vs. sham group; ${ }^{~} \mathrm{P}<0.05$ vs. IRI group. NLAG, $\mathrm{N}(2)-\mathrm{L}$-alanyl-L-glutamine; IRI, ischemia-reperfusion injury; LVDP, left ventricular end-diastolic pressure; $\mathrm{HR}$, heart rate; $+\mathrm{dP} / \mathrm{dtmax}$, maximum rate of left ventricular pressure.

fatty acid binding protein (hFABP; cat. no. SEB243Ra; Uscn Life Sciences, Inc.)], inflammatory cytokines [interleukin (IL) 1, 6 (cat. nos. SEA563Ra and SEA079Ra, respectfully; Uscn Life Sciences, Inc.) and tumor necrosis factor (TNF)- $\alpha$ (cat. no. SEA133Ra; Uscn Life Sciences, Inc.)], and oxidative stress factors [malondialdehyde (MDA; cat. no. CEA597Ge; Uscn Life Sciences, Inc.) and sorbitol dehydrogenase (SDH; cat. no. SEB495Ra; Uscn Life Sciences, Inc.)] were detected by ELISA kits. The optical density (OD) at $450 \mathrm{~nm}$ was measured with microplate reader.

Western blot analysis. A total of $100 \mathrm{mg}$ myocardial tissue was homogenized (IKA T10; IKA ${ }^{\circledR}$-Werke GmbH \& Co. KG, Breisgau, Germany) and centrifuged at $12,000 \mathrm{x}$ g for $15 \mathrm{~min}$ at $4^{\circ} \mathrm{C}$. Subsequently, the supernatant was collected and protein quantification was performed by bicinchoninic acid assay, and equal amounts of protein lysate $(40 \mu \mathrm{g})$ were separated by $12 \%$ SDS-PAGE. Transfer to nitrocellulose membranes was performed in transfer buffer $(12 \mathrm{mM}$ Tris base, $96 \mathrm{mM}$ glycine, $\mathrm{pH} 8.3$, and $15 \%$ methanol). Subsequently, the membranes were blocked for $2 \mathrm{~h}$ at room temperature in TBS with 20\% Tween 20 (TBST) buffer and probed with rabbit anti rat/human/mouse/cow B-cell lyphoma (Bcl)-2 (1:1,000; cat. no. ab59348; Abcam, Cambridge, UK), Bcl-associated X protein (Bax; 1:1,000; cat. no. ab32503; Abcam), Caspase-3 (1:500; cat. no. ab13847; Abcam), Janus kinase (JAK2; 1:5,000; cat. no. ab108596; Abcam), phosphorylated (p)-JAK2 (1:1,000; cat. no. ab32101; Abcam), signal transducer and activator of transcription (STAT) 3 (1:1,000; cat. no. ab68153; Abcam), p-STAT3 antibodies (1:2,000; cat. no. ab76315; Abcam), and a rabbit anti rat/human/mouse/monkey $\beta$-actin antibody (monoclonal; 1:100; cat. no. 8457; Cell Signaling Technology Inc., Danvers, USA) overnight at $4^{\circ} \mathrm{C}$. The membranes were washed with TBST buffer three times, followed by incubation with a horseradish peroxidase-conjugated secondary antibody (monoclonal; 1:4,000; cat. no. HS101; Beijing Transgen Biotech Co., Ltd., Beijing, China) for $1 \mathrm{~h}$ at room temperature. Finally, the protein expression levels were detected by using a Gel Doc $^{\text {TM }}$ EZ System (cat. no. 1708270; Bio-Rad Laboratories, Inc., Hercules, CA, USA), and densitometric analysis was then performed using Image $\mathrm{Lab}^{\mathrm{TM}}$ software (version 6.0; Bio-Rad Laboratories, Inc.).

Statistical analysis. All data are expressed as the mean \pm standard deviation. Analysis was performed using the GraphPad Prism software, version 6.00 (GraphPad Software Inc., La Jolla, CA, USA). Multiple comparisons were analyzed using

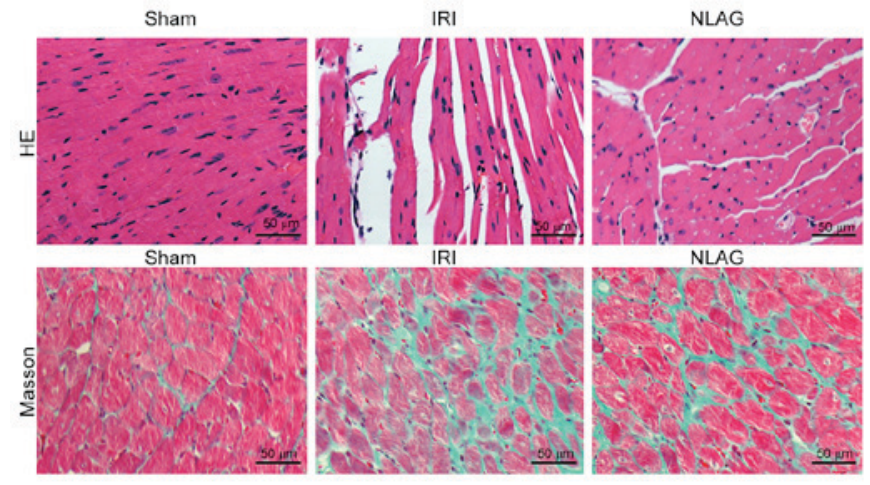

Figure 2. Effect of NLAG on the collagen deposition in the rat heart, as assessed by Masson and HE staining. NLAG, N(2)-L-alanyl-L-glutamine; IRI, ischemia-reperfusion injury; HE, Hematoxylin \& Eosin.

one-way analysis of variance followed by the Newman-Keuls post-hoc test. $\mathrm{P}<0.05$ was considered to indicate a statistically significant difference.

\section{Results}

EffectofNLAGonhemodynamicparameters.Compared withthe sham group (LVDP, HR and +dP/dtmax were $73.5 \pm 5.13 \mathrm{mmHg}$, $319.47 \pm 6.21$ beats $/ \mathrm{min}$ and $2,412.52 \pm 89.63 \mathrm{mmHg} / \mathrm{sec}$, respectively), LVDP, HR and +dP/dtmax were significantly decreased at 60 min following reperfusion in the IRI group (LVDP, HR and $+\mathrm{dP} / \mathrm{dtmax}$ were $31.3 \pm 4.53 \mathrm{mmHg}, 239.17 \pm 8.45$ beats $/ \mathrm{min}$ and $615.17 \pm 98.23 \mathrm{mmHg} / \mathrm{sec}$, respectively; P<0.05, Fig. 1). Furthermore,NLAGsignificantlyimprovedtheselevels(LVDP,HR and $+\mathrm{dP} / \mathrm{dtmax}$ were $57.81 \pm 3.46 \mathrm{mmHg}, 261.38 \pm 7.54$ beats $/ \mathrm{min}$ and $1320.15 \pm 110.12 \mathrm{mmHg} / \mathrm{sec}$, respectively; P<0.05, Fig. 1).

NLAG inhibits IRI-induced collagen deposition in the heart. Myocardial cells were arranged orderly in the Sham group, with clear boundaries and intact nuclei. On the contrary, myocardial cells in the IRI group were arranged disorderly, with unclear boundaries, myofiber rupture and nuclei disappearance. In the NLAG group, the severity of myocardial injuries was improved to a great extent (Fig. 2).

NLAG modulates IRI-induced protein expression of cardiac enzymes. Compared with the Sham group, the protein levels of LDH, cTnI, CK and hFABP in the plasma of the IRI group were increased significantly $(\mathrm{P}<0.05)$. On the other hand, NLAG effectively inhibited their expression levels, which was 

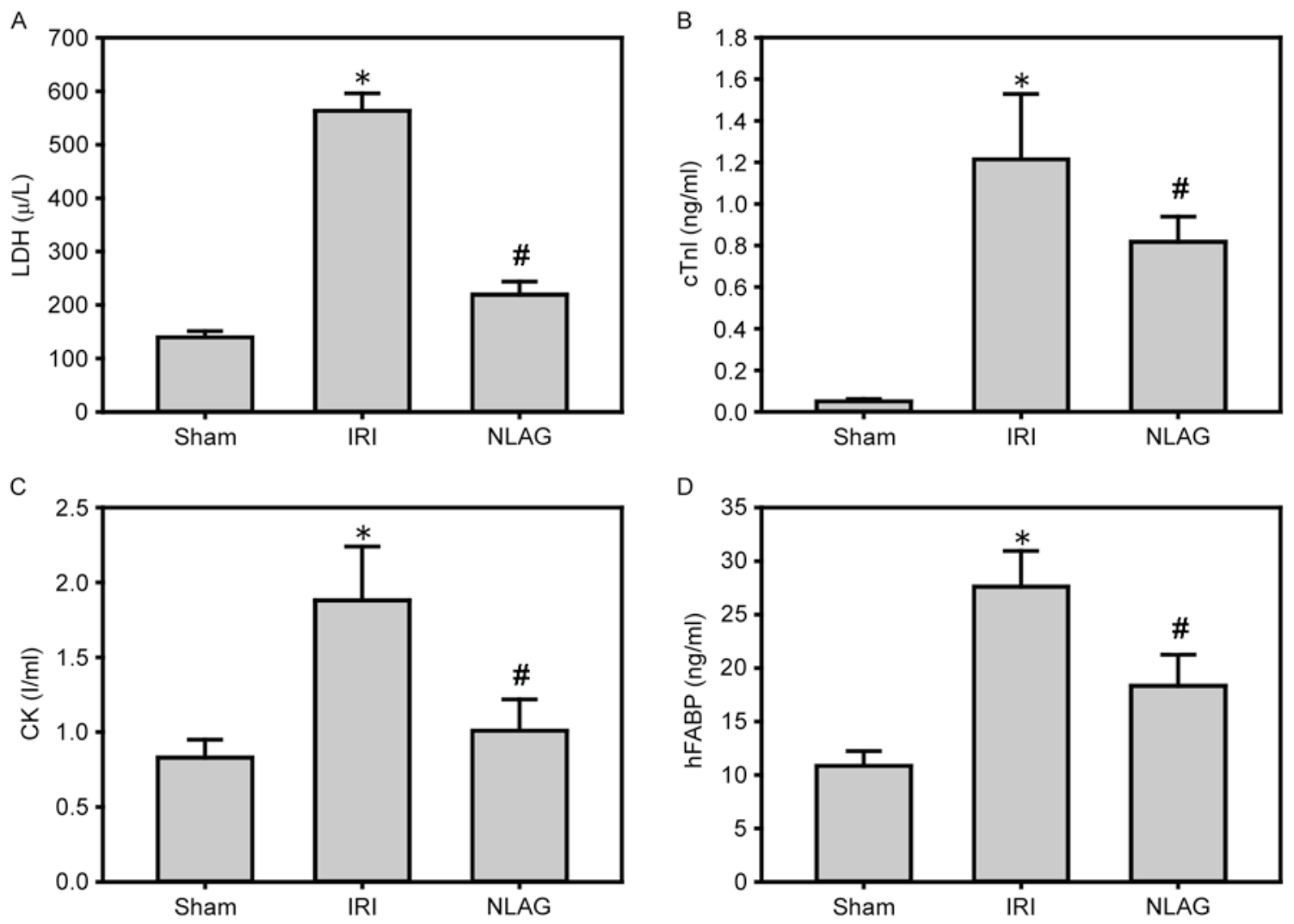

Figure 3. Effect of NLAG on cardiac enzymes in rats, assessed by ELISA. (A) LDH, (B) cTnI, (C) CK and (D) hFABP. Data are presented as the mean \pm standard deviation. "P<0.05 vs. sham group; ${ }^{\#} \mathrm{P}<0.05$ vs. IRI group. NLAG, N(2)-L-alanyl-L-glutamine; IRI, ischemia-reperfusion injury; LDH, lactase dehydrogenase; cTnI, troponin I; CK, creatine kinase; hFABP, heart type fatty acid binding protein.
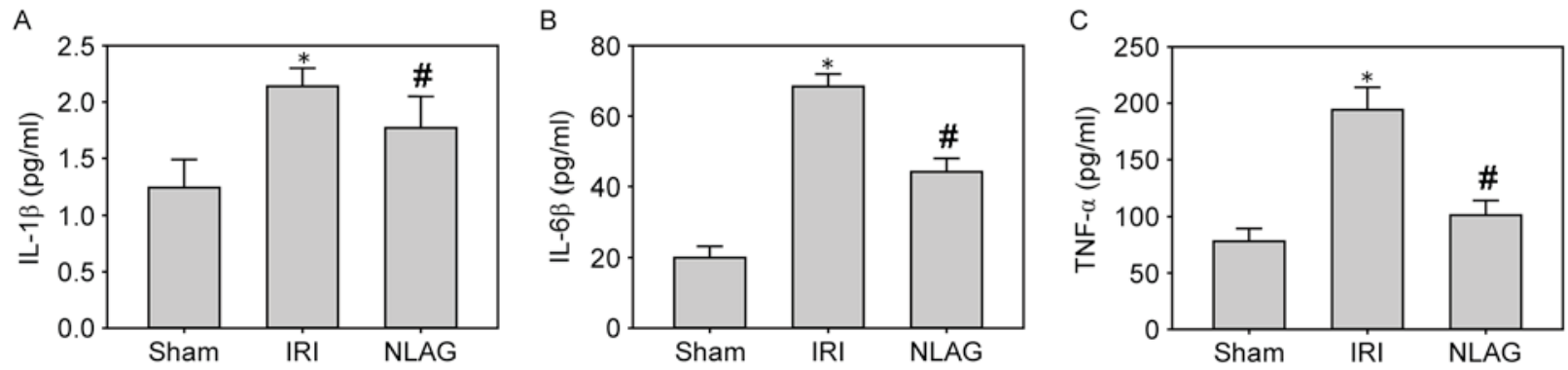

Figure 4. Effect of NLAG on inflammatory cytokines in rats, assessed by ELISA. (A) IL-1 $\beta$, (B) IL-6 $\beta$ and (C) TNF- $\alpha$ levels. Data are presented as the mean \pm standard deviation. "P $<0.05$ vs. sham group; ${ }^{*} \mathrm{P}<0.05$ vs. IRI group. NLAG, $\mathrm{N}(2)$-L-alanyl-L-glutamine; IRI, ischemia-reperfusion injury; IL, interleukin; TNF, tumor necrosis factor.

statistically significant compared with the IRI group $(\mathrm{P}<0.05$, Fig. 3).

NLAG modulates IRI-induced protein expression of inflammatory cytokines. Compared with the Sham group, the protein levels of IL-1 $\beta$, IL- 6 and TNF- $\alpha$ in the plasma of the IRI group were increased significantly $(\mathrm{P}<0.05)$. However, NLAG could effectively inhibit the inflammatory response, which was statistically significant compared with the IRI group ( $\mathrm{P}<0.05$, Fig. 4$)$.

NLAG inhibits IRI-induced protein expression of oxidative stress. Compared with the Sham group, the protein expression levels of MDA and SDH were increased in the IRI group
$(\mathrm{P}<0.05)$. However, NLAG effectively inhibited the expression of the oxidative stress indicators, which was statistically significant compared with the IRI group $(\mathrm{P}<0.05$, Fig. 5).

NLAG modulates IRI-induced protein expression of Bcl-2, Bax and Caspase-3. Compared with the Sham group, the protein expression levels of Bax and Caspase-3 in the IRI group were increased and the expression of Bcl-2 was decreased, which was significant $(\mathrm{P}<0.05)$. On the contrary, NLAG significantly increased Bcl-2 expression, and decreased Bax and Caspase-3 expression compared with the IRI group $(\mathrm{P}<0.05$, Fig 6).

NLAG modulates IRI induced-protein expression of JAK2, $p$-JAK2, STAT3 and p-STAT3. Compared with the Sham 

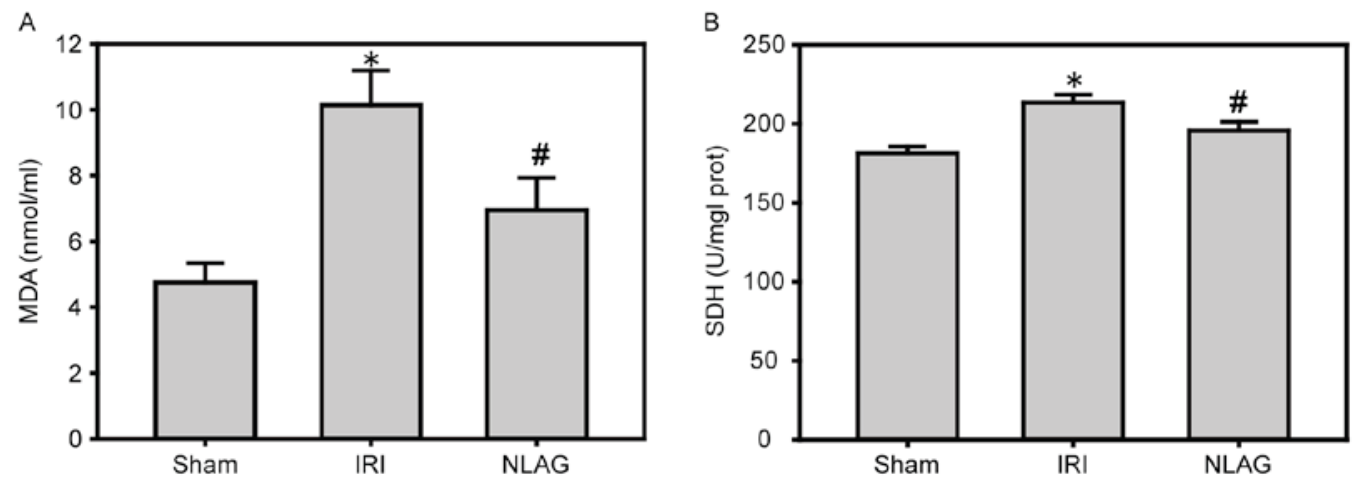

Figure 5. Effect of NLAG on oxidative stress in the rats, assessed by ELISA. (A) MDA and (B) SDH levels. Data are presented as the mean \pm standard deviation. "P<0.05 vs. sham group; ${ }^{*} \mathrm{P}<0.05$ vs. IRI group. NLAG, N(2)-L-alanyl-L-glutamine; IRI, ischemia-reperfusion injury; MDA, malondialdehyde; SDH, succinate dehydrogenase.

A

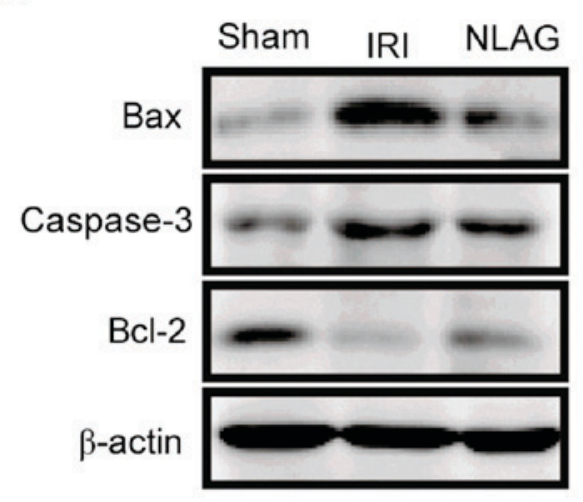

B

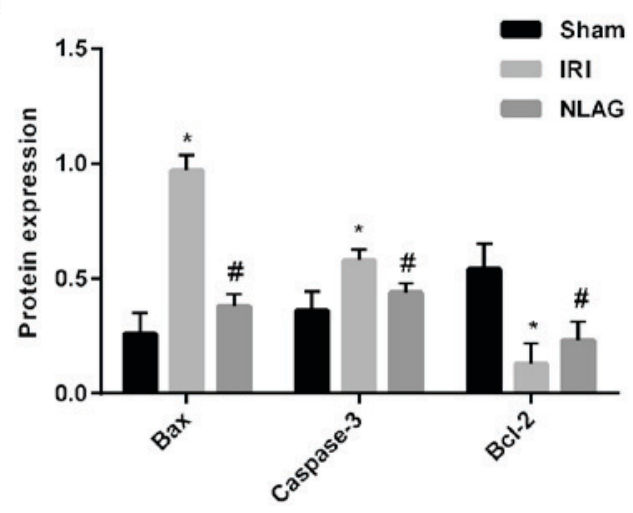

Figure 6. Expression levels of Bax, Caspase-3 and Bcl-2 proteins were detected by western blot assay. (A) Western blot analysis of Bax, Bcl-2 and Caspase-3. (B) Protein expression of Bax, Caspase- 3 and Bcl-2 gene expression. Data are presented as the mean \pm standard deviation. ${ }^{*} \mathrm{P}<0.05$ vs. sham group; ${ }^{\#} \mathrm{P}<0.05$ vs. IRI group. NLAG, N(2)-L-alanyl-L-glutamine; IRI, ischemia-reperfusion injury; Bcl, B-cell lymphoma; Bax, Bcl-associated X protein.

A

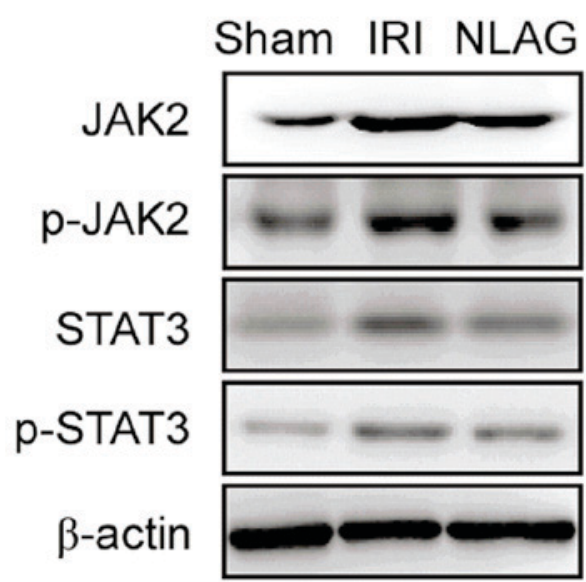

B

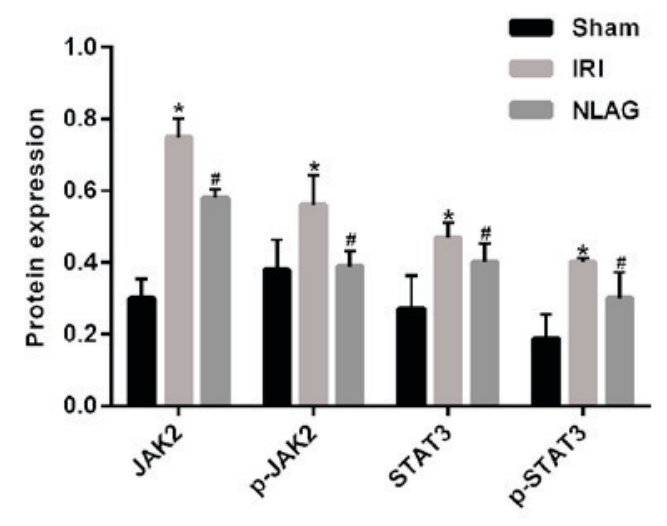

Figure 7. Expression levels of JAK2, p-JAK2, STAT3, p-STAT3 proteins were detected by western blot assay. (A) Western blot analysis of JAK2, p-JAK2, STAT3 and p-STAT3 protein expression levels. (B) Protein levels of JAK2, p-JAK2, STAT3 and p-STAT3. Data are presented as the mean \pm standard deviation. " $\mathrm{P}<0.05$ vs. sham group; ${ }^{*} \mathrm{P}<0.05$ vs. IRI group. NLAG, N(2)-L-alanyl-L-glutamine; IRI, ischemia-reperfusion injury; JAK, Janus kinase; STAT, Signal transducer and activator of transcription; $\mathrm{p}$, phosphorylated.

group, the protein expression levels of JAK2, p-JAK2, STAT3 and p-STAT3 were decreased significantly in the IRI group $(\mathrm{P}<0.05)$. On the other hand, NLAG significantly increased JAK2, p-JAK2, STAT3 and p-STAT3 expression compared with the IRI group $(\mathrm{P}<0.05$, Fig. 7).

\section{Discussion}

In the present study, a rat IRI model was used to explore the effects of NLAG on IRI and observe the histopathological changes and protein expression changes of inflammatory 
cytokines (such as IL-1 $\beta$, IL-6 and TNF- $\alpha$ ), of oxidative stress markers (such as MDA and SDH), of apoptosis-associated proteins (such as Bcl-2, Bax and Caspase-3) and of the JAK2/STAT3 signaling pathway-associated proteins. It was demonstrated that NLAG could reduce the IRI-induced injury on cardiomyocytes, downregulate the expression of inflammatory factors, and decrease the apoptosis to some extent. In addition, NLAG prevented IRI-induced myocardial injury and inhibited JAK2, STAT3, p-STAT3 and p-JAK2 expression. This study was aimed to investigate the effects of NLAG on myocardial injury and to explore the regulatory mechanism of JAK2/STAT3 signaling pathway on myocardial injury, which provides a basis for cardiopulmonary bypass.

Previous studies have demonstrated that IRI induces death mechanisms in a variety of cells, including apoptosis, necrosis and autophagy $(15,16)$. The STAT protein family serves an important role in the regulation of cardiomyocyte apoptosis, whereas STAT3 serves a protective role. Boengler et al (17) identified that myocardial infarcted areas were greater in the STAT3-knockout mice following IRI treatment than in the wild-type mice, with more apoptotic cardiomyocytes and higher mortality. In addition, cardiac-specific STAT3 knockout is more sensitive to inflammatory injury, as the IRI-induced inflammation was more obvious in the STAT3 knockout mice, especially following myocardial necrosis. The JAK2/STAT3 signaling pathway is an important component of the survivor activating factor enhancement, which protects against the reperfusion injury through activation of TNF- $\alpha$ (18). Butler et al (19) have demonstrated that blood supply from an ischemic preconditioning heart to another heart can activate STAT3 in the recipient protecting the myocardial tissue. In the present study, the rat IRI model exhibited myocardial damage, disordered arrangement of myocardial cells and high levels of LDH release, inflammatory factors (IL-1 $\beta$, IL-6 and TNF- $\alpha$ ) and oxygen-free radicals (MDA and SDH), increased expression of apoptosis-associated proteins, and decreased expression of JAK2/STAT3-associated proteins. These results suggest that IRI can induce myocardial injury through inflammation and apoptosis and the JAK2/STAT3 signaling pathway serves an important role in inflammation, oxidative stress, cell injury and apoptosis. Terrell et al (20) have demonstrated that IL-6 stimulates cardiac hypertrophy through activation of the JAK/STAT signaling pathway. Park et al (21) presented that the JAK/STAT signaling pathway mediates oxidative stress-induced reduction of pulmonary surfactant epithelium synthesis. In the present study, inhibition of the JAK/STAT signaling pathway reduced the IRI-induced myocardial injury and apoptosis. Li et al (22) identified that fasudil exerts its anti-IRI effect by inhibiting the endoplasmic reticulum stress response and by activating the JAK2/STAT3 signaling pathway. Luan et al (23) demonstrated that hydrogen sulfide post-treatment can activate the JAK2/STAT3 signaling pathway, thus protecting the isolated heart from myocardial IRI. According to the study of Dong et al (24), rapamycin can exert its anti-IRI action in vivo and in vitro by activating the JAK2/STAT3 signaling pathway. It has been widely used in the treatment of respiratory diseases and digestion systemic diseases. NLAG also improves ventricular remodeling and reduces myocardial damage. In the present study, NLAG inhibited both inflammatory factors and oxidative stress indicators, activated JAK2 and STAT3 proteins, and significantly increased the expression of p-JAK2 and p-STAT3. These findings indicate that NLAG can attenuate myocardial IRI, and that the JAK2/STAT3 signaling pathway serves an important regulatory role in that process.

In conclusion, the results of the present study suggest that myocardial ischemia-reperfusion may lead to myocardial cell apoptosis and myocardial injury, and that NLAG attenuates IRI-induced mitochondrial oxidative stress injury and apoptosis via activation of the JAK2/STAT3 signaling pathway, thus exerting protective effects against IRI.

\section{Acknowledgements}

The present study was supported by the Tianjin Municipal Health and Family Planning Commission of Science and Technology projects (grant no. 14KG125).

\section{References}

1. Igarashi M, Tada H, Yamasaki H, Kuroki K, Ishizu T, Seo Y, Machino T, Murakoshi N, Sekiguchi Y, Noguchi Y, et al: Fragmented QRS is a novel risk factor for ventricular arrhythmic events after receiving cardiac resynchronization therapy in non-ischemic cardiomyopathy. J Cardiovasc Electrophysiol 28: 327-335, 2017.

2. Cordeiro B and Clements R: Murine isolated heart model of myocardial stunning associated with cardioplegic arrest. J Vis Exp: e52433, 2015.

3. Wong GW, Laugerotte A and Wright JM: Blood pressure lowering efficacy of dual alpha and beta blockers for primary hypertension. Cochrane Database Syst Rev: CD007449, 2015.

4. Devi NR, Sumitra C and Singh NR: Absorption spectral study of $\operatorname{Pr}(\mathrm{III})$ complexes with L-Alanyl-L-Glutamine and N-Acetyl-L-Glutamine: Interaction parameters, bonding and Judd-Ofelt intensity. Asian J Chem 24: 2863-2870, 2012.

5. Cunha Filho JF, Gonçalves II, Guimarães SB, Jamacaru FV, Garcia JH and Vasconcelos PR: L-alanyl-glutamine pretreatment attenuates acute inflammatory response in children submitted to palatoplasty. Acta Cir Bras 26 (Suppl 1): S72-S76, 2011.

6. Gabr A, Salem AAS, Samy HA, Tmam S and Ali AM: N(2)-L-Alanyl-L-Glutamine dipeptide preventing oxaliplatin-induced neurotoxicity in colorectal cancer patients 07 : 609-621, 2016.

7. Pires VL, Souza JR, Guimarães SB, Silva Filho AR, Garcia JH and Vasconcelos PR: Preconditioning with L-alanyl-L-glutamine in a Mongolian gerbil model of acute cerebral ischemia/reperfusion injury. Acta Cir Bras 26 (Suppl 1): S14-S20, 2011.

8. Araújo Júnior RJ, Silva Júnior RG, Vasconcelos MP, Guimarães SB, Vasconcelos PR and Garcia JH: Preconditioning with L-alanyl-glutamine reduces hepatic ischemia-reperfusion injury in rats. Acta Cir Bras 26 (Suppl 1): S8-S13, 2011.

9. Shen-Orr SS, Furman D, Kidd BA, Hadad F, Lovelace P, Huang YW, Rosenberg-Hasson Y, Mackey S, Grisar FA, Pickman Y, et al: Defective signaling in the JAK-STAT pathway tracks with chronic inflammation and cardiovascular risk in aging humans. Cell Syst 3: 374-384.e4, 2016.

10. De S, Manna A, Kundu S, De Sarkar S, Chatterjee U, Sen T, Chattopadhyay S and Chatterjee M: Allylpyrocatechol attenuates collagen-induced arthritis via attenuation of oxidative stress secondary to modulation of the MAPK, JAK/STAT, and Nrf2/HO-1 pathways. J Pharmacol Exp Ther 360: 249-259, 2017.

11. Kim HC, Kim E, Bae JI, Lee KH, Jeon YT, Hwang JW, Lim YJ, Min SW and Park HP: Sevoflurane postconditioning reduces apoptosis by activating the JAK-STAT pathway after transient global cerebral ischemia in rats. J Neurosurg Anesthesiol 29: 37-45, 2017.

12. Mudaliar H, Rayner B, Billah M, Lay W and Bhindi R: Remote ischaemic preconditioning activates the JAK-STAT pathway resulting in the attenuation of Egr-1 expression following myocardial ischaemia reperfusion injury. Heart Lung Circulat 24 (Suppl 3): S163, 2015. 
13. Wen SH, Li Y, Li C, Xia ZQ, Liu WF, Zhang XY, Lei WL, Huang WQ and Liu KX: Ischemic postconditioning during reperfusion attenuates intestinal injury and mucosal cell apoptosis by inhibiting JAK/STAT signaling activation. Shock 38: 411-419, 2012.

14. Baguisi A, Casale RA, Kates SA, Lader AS, Stewart K and Beeuwkes R III: CMX-2043 efficacy in a rat model of cardiac ischemia-reperfusion injury. J Cardiovasc Pharmacol Ther 21: 563-569, 2016.

15. Shu S, Li CM, You YL, Qian XL, Zhou S and Ling CQ: Electroacupuncture ameliorates cerebral ischemia-reperfusion injury by regulation of autophagy and apoptosis. Evid Based Complement Alternat Med 2016: 7297425, 2016.

16. Wang Z, Yu J, Wu J, Qi F, Wang H, Wang Z and Xu Z: Scutellarin protects cardiomyocyte ischemia-reperfusion injury by reducing apoptosis and oxidative stress. Life Sci 157: 200-207, 2016.

17. Boengler K, Buechert A, Heinen Y, Roeskes C, Hilfiker-Kleiner D, Heusch G and Schulz R: Cardioprotection by ischemic postconditioning is lost in aged and STAT3-deficient mice. Circ Res 102: 131-135, 2008.

18. Jacoby JJ, Kalinowski A, Liu MG, Zhang SS, Gao Q, Chai GX, Ji L, Iwamoto Y, Li E, Schneider M, et al: Cardiomyocyte-restricted knockout of STAT3 results in higher sensitivity to inflammation, cardiac fibrosis, and heart failure with advanced age. Proc Natl Acad Sci USA 100: 12929-12934, 2003.

19. Butler KL, Huffman LC, Koch SE, Hahn HS and Gwathmey JK: STAT-3 activation is necessary for ischemic preconditioning in hypertrophied myocardium. Am J Physiol Heart Circ Physiol 291: H797-H803, 2006.
20. Terrell AM, Crisostomo PR, Wairiuko GM, Wang M, Morrell ED and Meldrum DR: Jak/STAT/SOCS signaling circuits and associated cytokine-mediated inflammation and hypertrophy in the heart. Shock 26: 226-234, 2006.

21. Park SK, Dahmer MK and Quasney MW: MAPK and JAK-STAT signaling pathways are involved in the oxidative stress-induced decrease in expression of surfactant protein genes. Cell Physiol Biochem 30: 334-346, 2012.

22. Li Y, Zhu W, Tao J, Xin P, Liu M, Li J and Wei M: Fasudil protects the heart against ischemia-reperfusion injury by attenuating endoplasmic reticulum stress and modulating SERCA activity: The differential role for PI3K/Akt and JAK2/STAT3 signaling pathways. PLoS One 7: e48115, 2012.

23. Luan HF, Zhen LI, Zhao QH, Wang L, Yong JI and Zeng YM: Role of JAK2/STAT3 signaling pathway in hydrogen sulfide postconditioning on isolated ischemia/reperfusion rat hearts. Chin J Pharmacol Toxicol 25: 23-28, 2011 (In Chinese).

24. Dong J, Jiang Y, Liu A, Wang S, Yang Q and Zhang Y: Expression of JAK/STAT signal transduction pathway and the effects of rapamycin in lupus nephritis-prone MRL/lpr mice. Chin J Microbiol Immunol 15: 73-77, 2007.

This work is licensed under a Creative Commons Attribution-NonCommercial-NoDerivatives 4.0 International (CC BY-NC-ND 4.0) License. 